\title{
INFLUENCE OF NANO ALUMINIUM POWDER PRODUCED BY WIRE EXPLOSION PROCESS AT DIFFERENT AMBIENCE ON HYDROGEN GENERATION
}

\author{
Ramanujam Sarathi ${ }^{*}$ - Binu Sankar* \\ - Satyanarayanan R. Chakravarthy ${ }^{* *}$
}

\begin{abstract}
Nano-aluminium particles are produced through the wire explosion process in different gas medium. The particles produced by wire explosion process, in helium medium are of smaller size compared to argon/nitrogen medium. The nano aluminium powder on reaction with water forms oxides having bayerite and boehmite structure. It is observed that nano aluminium on reaction with $\mathrm{KOH}$ solution at room temperature it forms bayerite. The results of the study were confirmed through Wide Angle X-ray diffraction (WAXD) and by Transmission Electron Microscope (TEM) studies. The reaction of nano aluminium powder with $\mathrm{KOH}$ solution/water indicates that the rate of hydrogen generation is high when nano aluminium powder reacts with $\mathrm{KOH}$ solution than with water. The rate of hydrogen generation gets reduced drastically when the nano aluminium powder which is exposed to air medium for some period is used for reaction with $\mathrm{KOH} /$ water. It is also observed that the rate of hydrogen generation is high with nano size aluminium particles compared with ultrafine particles.
\end{abstract}

K e y w ord s: nano aluminium, wire explosion, hydrogen generation, WAXD, TEM

\section{INTRODUCTION}

The development of new type of generation of hydrogen sources to meet the drastically growing global energy demand is one of the major challenges to industries and researchers in the world. Methodology of generation of hydrogen has to be established so that the technology can be implemented for any use in a sustainable, clean, user friendly, safe, reliable and by economic means. Conventional method of generation of hydrogen involves biological process, electrochemical, water electrolysis or by chemical methods and every process have certain limitations $[1,2]$. Aluminium and its alloys are recognised to be some of the most reliable metal applicable for hydrogen production $[3,4]$. Considerable work has been carried out on understanding the generation of hydrogen gas by metal reaction with alkali solution [5]. It is realised that size and shape of the particle play a predominant role on the rate of generation of hydrogen gas.

Having known all this, in the present work, the nano aluminium powder is produced by wire explosion process in different ambiences and a methodical experimental study was carried out to understand the influence of size of nano aluminium particle on hydrogen generation through metal reaction with alkali and water. For the purpose of comparison, hydrogen generation processes were also carried out using ultra fine aluminium particles. Wide Angle X-ray diffraction (WAXD) studies were carried out to understand the characteristics of nano aluminium powder and on reaction with water and alkali so- lution. The size and shape of the nano aluminium powder and the precipitates formed on reaction with water/alkali solution were analysed using WAXD studies and by TEM analysis. In addition the rate of hydrogen generation on reaction of nano particles of different sizes produced in different inert atmospheres through Wire Explosion Process (WEP) with water/alkali solution were measured and analysed.

\section{EXPERIMENTAL STUDIES}

In the present work, wire explosion technique is adopted, which is a top-down approach to produce nano powders. This is basically an evaporation technique, where the particles are produced by evaporating a thin metal conductor by passing high current through it, in an inert atmosphere [6-8]. In this technique, by providing appropriate energy to the conductor to evaporate, and by maintaining a suitable medium, it is possible to control the size and shape of the particle $[9,10]$. The wire explosion process was conducted at an operating pressure of 1 Atmosphere.

The basic circuit used for exploding the wire to form nano powders is shown in Fig. 1. The capacitor is charged to the required voltage magnitude through a voltagedoubler circuit. Upon closing the switch S (Fig. 1), the voltage appears across the wire and the current (controlled by the RLC circuit) rises, causing Joule-heating of the conductor, which eventually melts [11]. In the circuit, switch $\mathrm{S}$ is a high-voltage trigatron gap, $R$ is the

\footnotetext{
${ }^{*}$ Department of Electrical Engineering, ${ }^{* *}$ Department of Aerospace Engineering, Indian Institute of Technology Madras, Chennai, India, rsarathi@iitm.ac.in
} 


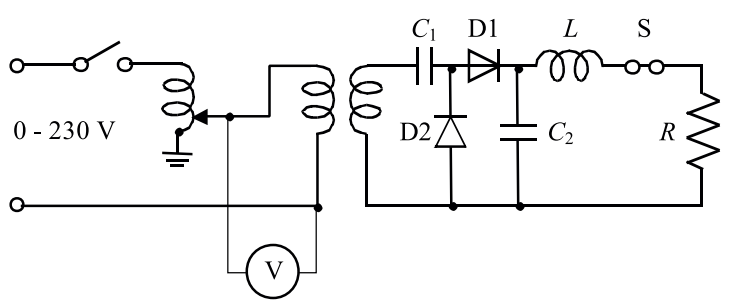

Fig. 1. Basic circuit in the wire explosion experimental set-up

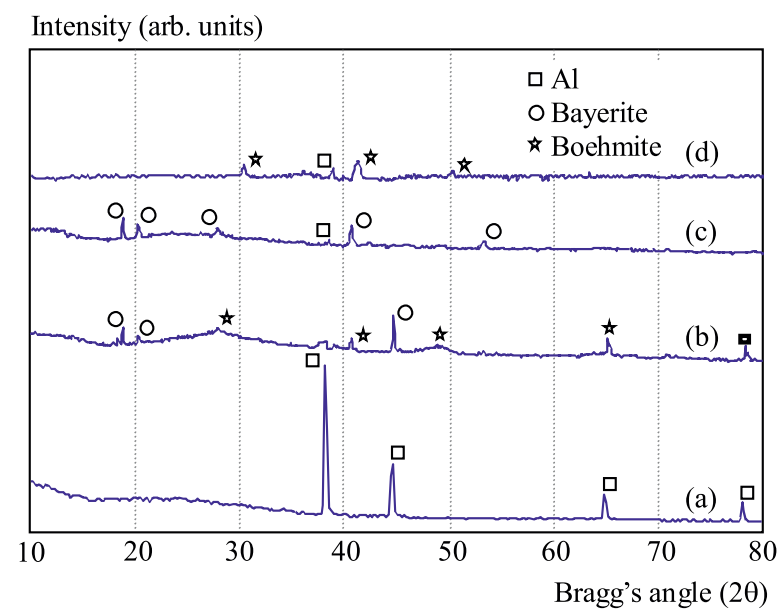

Fig. 2. Typical XRD analysis of (a) nano aluminium produced by WEP in Helium ambience (b) on reaction with water $(\mathrm{PH}=7)$ (c) $0.5 \mathrm{M} \mathrm{KOH}(\mathrm{PH}=12)(\mathrm{d}) 0.5 \mathrm{M} \mathrm{KOH}$ solution maintained at $50{ }^{\circ} \mathrm{C}$

Table 1. Summary of Experimental Details

\begin{tabular}{ll}
\hline Capacitance & $3 \mu \mathrm{F}$ \\
Charging Voltage & $25 \mathrm{kV}$ \\
Material & Aluminium \\
Wire diameter & $0.5 \mathrm{~mm}$ \\
Length of the wire & $150 \mathrm{~mm}$ \\
Ambience & Argon, Helium and Nitrogen \\
\hline
\end{tabular}

exploding wire resistance, and $L$ is the contribution by the internal inductance of the capacitor and the lead inductance and forms RLC circuit [12]. The dimension of the exploding wire used in the present study, values of the capacitances and the charging voltage, the different ambience used during wire explosion are provided in Table 1 . In the present study, the nano aluminium particles have been produced in helium, argon and nitrogen ambiences. The wire explosion was conducted at 1 atmospheric pressure.

In the present study, nano aluminium powders were produced by wire explosion process and its reactions were studied in water and alkali medium viz. neutral medium with $\mathrm{PH}$ value of 7 and base medium with $\mathrm{PH}$ value of 12 (by $0.5 \mathrm{M} \mathrm{KOH}$ ). A $25 \mathrm{mg}$ of nano aluminium powder mixed with $5 \mathrm{ml}$ of alkali base/neutral medium and the evolved gas was connected to a calibrated manometer to measure the quantity of hydrogen evolved in the process.
The measurements were carried out until the process is complete. To understand the impact of size of aluminium particle on generation of hydrogen, commercially available micron size aluminium powder was added to the alkaline solution and the rate of hydrogen generation were also measured.

The chemical reaction governing the reaction of nano aluminium [13] with alkaline solution and pure water are given as

$$
\begin{aligned}
2 \mathrm{Al}+2 \mathrm{KOH}+6 \mathrm{H}_{2} \mathrm{O} & \rightarrow 2 \mathrm{Al}(\mathrm{OH})_{3}+2 \mathrm{KOH}+3 \mathrm{H}_{2} \uparrow \\
2 \mathrm{Al}+6 \mathrm{H}_{3} \mathrm{O} & \rightarrow 2 \mathrm{Al}(\mathrm{OH})_{3}+3 \mathrm{H}_{2} \uparrow \\
\mathrm{Al}(\mathrm{OH})_{3} & \rightarrow \mathrm{AlOOH}+\mathrm{H}_{2} \mathrm{O}
\end{aligned}
$$

The number of moles of hydrogen liberated due to reaction of aluminium powder with alkali base/ water can be calculated as follows.

The number of moles of evolved hydrogen $n \mathrm{H}_{2}(t)$ liberated in the reaction process could be calculated using the ideal gas law. At the instant of start of reaction at time $t=0$, the ideal gas law equation could be written as

$$
P_{0} V_{0}=n_{\text {air }} R T
$$

where $P_{0}=P_{\text {atm }}$ and $V_{0}=(100 \pm 20) \mathrm{ml}$ is the volume of the air in the reactor-flask system, $R$ is gas constant and $T$ is the temperature in degree Kelvin.

On generation of gaseous hydrogen the fundamental gas equation can be written as

$$
P(t) V(t)=\left(n_{\text {air }}+n \mathrm{H}_{2}(t)\right) R T
$$

where $V(t)$ is given by the sum of the initial volume of air $\left(V_{0}\right)$ and the volume of water displaced due to liberated hydrogen $(v(t))$.

Combining equations 4 and 5 the number of moles of hydrogen evolved could be estimated as

$$
n \mathrm{H}_{2}(t)=\frac{P(t) V(t)}{R T}-\frac{P_{0} V_{0}}{R T}
$$

The rate of hydrogen generation is more important in this process. This could be calculated as a function of the controlled variables as

$$
\% \text { Yield } \mathrm{H}_{2}=\frac{n \mathrm{H}_{2}(t)}{n \mathrm{H}_{2} \mathrm{O}} \times 100
$$

where $n \mathrm{H}_{2} \mathrm{O}$ is the number of moles of water added, and

$$
\text { Rate } \mathrm{H}_{2}=\frac{\mathrm{d} v(t)}{\mathrm{d} t}
$$

Rate $\mathrm{H}_{2}$ is the rate of evolved hydrogen in millilitres per minute and grams of aluminium. 

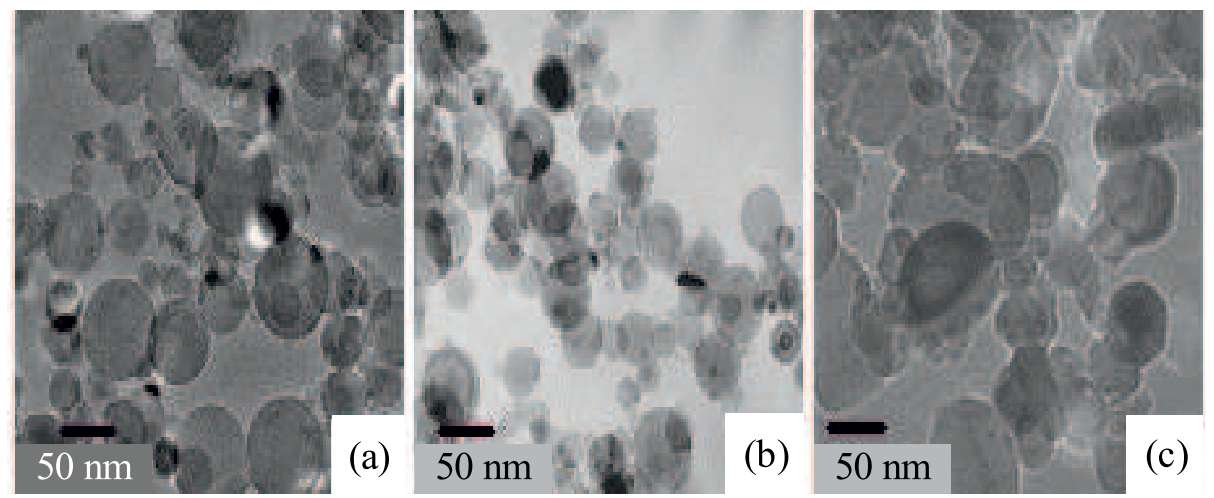

Fig. 3. TEM images of nano aluminium particles obtained in different ambiences (a) argon (b) helium (c) nitrogen

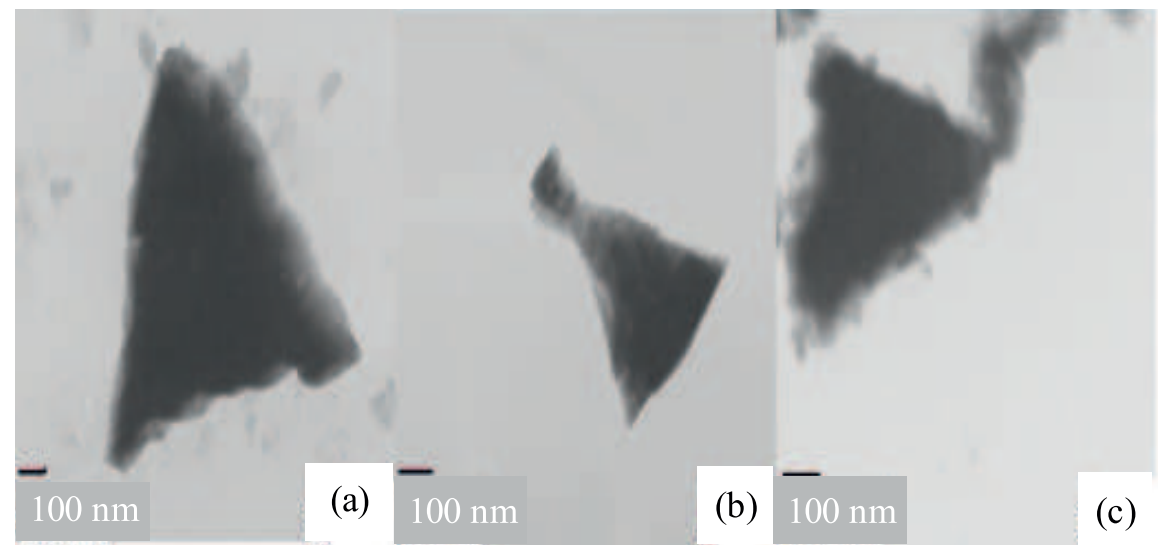

Fig. 4. Typical TEM pattern of bayerite formed when nano aluminium generated in different ambience reacts with $0.5 \mathrm{M} \mathrm{KOH}$ (a) helium (b) argon (c) nitrogen

Table 2. Median diameter and standard deviation

\begin{tabular}{ccc}
\hline Medium & $\begin{array}{c}\text { Median } \\
\text { diameter } \\
\left(D_{50}, \mathrm{~nm}\right)\end{array}$ & $\begin{array}{c}\text { Geometric } \\
\text { Standard } \\
\text { deviation }\left(\sigma_{g}\right)\end{array}$ \\
\hline Helium & 33.1 & 1.93 \\
Argon & 41.0 & 1.65 \\
Nitrogen & 33.7 & 1.8 \\
\hline
\end{tabular}

\section{Physico-chemical diagnostic study}

Wide angle X-ray diffraction (WAXD) measurements were done using Phillips X-ray diffractometer. A scan rate of $2^{\circ} / \mathrm{min}$ at 2000 cycles using $\mathrm{CuK} \alpha$ radiation of wavelength $1.5426 \AA$ was applied. A radial scan of diffraction angle $(2 \theta)$ vs intensity was obtained with an accuracy of $\pm 0.25^{\circ}$ at the location of the peak. The shape and size measurements of the particles were carried out through transmission electron microscopy (TEM) studies (CM-12 Scanning transmission electron microscopy). The particle size was evaluated based on the TEM bright field images. The number of analyzed particle for each synthesis was around 500 .

\section{RESULTS AND DISCUSSION}

Figure 2 shows the WAXD spectra of the nanoaluminium powder formed by the wire explosion process in helium ambience and on its reaction with alkaline base and neutral medium. It is realized that the nano aluminium powder obtained in helium ambience have peaks at $38.5^{\circ}, 44.7^{\circ}, 65.1^{\circ}$ and $78.2^{\circ}$, which are the characteristic peaks that occur in the XRD pattern of the aluminium powder[14] and has not reflected the peaks of any oxides (Fig. 2a). In the present study, the hydrogen gas was generated through the reaction of nano aluminium with $\mathrm{KOH} /$ water solution. The precipitates formed in the reaction were analysed. The reaction of the nano aluminium with water formed precipitates were analysed through WAXD studies, which indicates the formation of oxides having structure of bayerite and boehmite (Fig. 2b) [15]. The nano aluminium powder on its reaction with $0.5 \mathrm{M} \mathrm{KOH}$ yields hydrogen gas and the precipitates of oxides having bayerite as the main phase (Fig. 2c).Similar reaction of nano aluminium with $0.5 \mathrm{M} \mathrm{KOH}$ maintained at $50^{\circ} \mathrm{C}$, the end product shows formation of oxides having both bayerite and boehmite structure.

Figure 3 shows the TEM structure of the nanoaluminium powder formed by the wire explosion process 

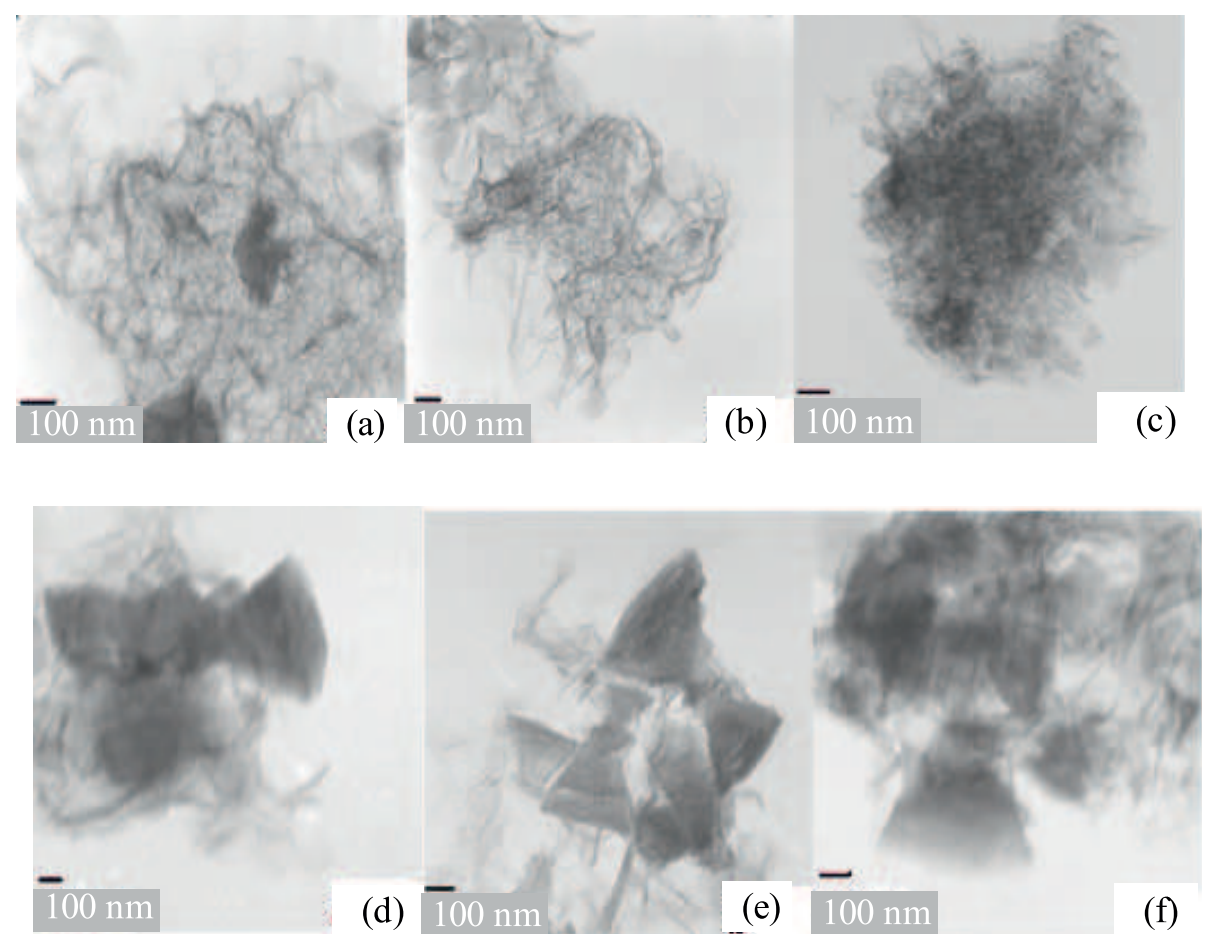

Fig. 5. Typical TEM pattern of boehmite: (a) helium (b) argon (c) nitrogen, and bayerite: (d) helium (e) argon (f) nitrogen formed due to reaction of nano aluminium powder produced in different ambience with water

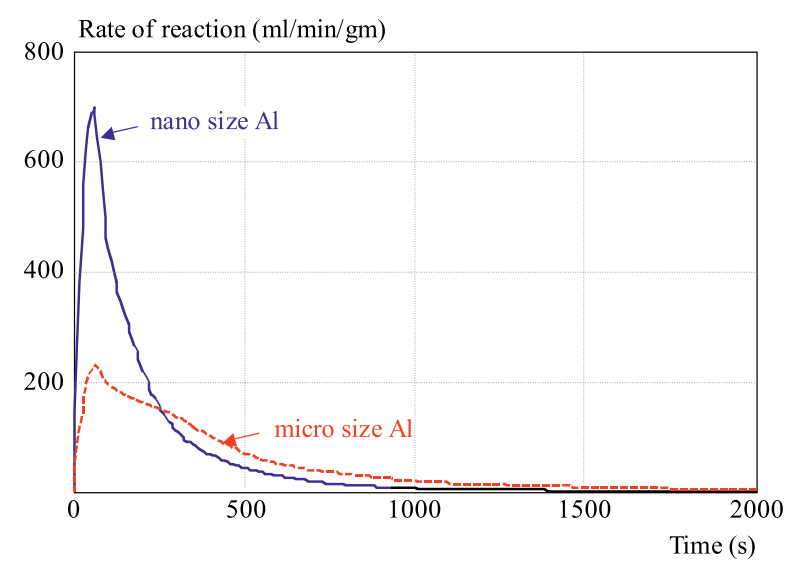

Fig. 6. Rate of reaction of micron and nano sized aluminium powder with $0.5 \mathrm{M} \mathrm{KOH}$

in helium, argon and nitrogen medium. It is observed that the aluminium particles produced (in argon and helium ambience) through wire explosion process is spherical in shape. The particle size lies in the range of a few nm up to $\sim 100 \mathrm{~nm}$. It is observed that the particle size measurement follows the log-normal distribution $[16,17]$. The geometric mean and standard deviation of the particles were calculated adopting log-normal studies and are provided in Table 2.

The particles produced in different atmospheres on its reaction with alkali base/water generates hydrogen and produces hydroxides as the reactions explained through equations 2 and 3. (bayerite and boehmite structures). When the nano aluminium particles react with $0.5 \mathrm{M} \mathrm{KOH}$ alkaline solution triangular shaped structures

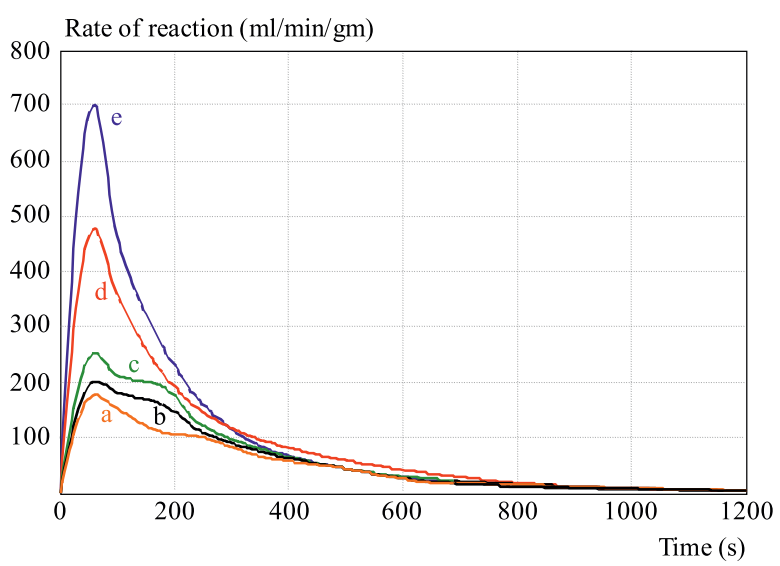

Fig. 7. Rate of reaction when nano aluminium reacted with different molarity of $\mathrm{KOH}$ (a) $0.1 \mathrm{M}$ (b) $0.2 \mathrm{M}$ (c) $0.3 \mathrm{M}$ (d) $0.4 \mathrm{M}$ and (e) $0.5 \mathrm{M}$

were formed which is the characteristic shape of bayerite. This is more predominant when nano aluminium powder reacts with alkali solution (Fig. 4). The TEM picture of nano aluminium powder on reaction with water indicates the presence of fibrillar structure (Fig. 5a,b,c which are boehmite structures) with few triangular typed structures (bayerite) (Fig. 5d,e,f).The structural analysis of the compound confirms the results obtained through WAXD studies.

Figure 6 shows the variation in rate of generation of hydrogen on reaction of micron sized and nano sized aluminium particles produced in helium ambience with $0.5 \mathrm{M} \mathrm{KOH}$ solution. It is observed that the reaction rate is high with nano aluminium powder compared with micron sized aluminium powder. This variation in the rate 

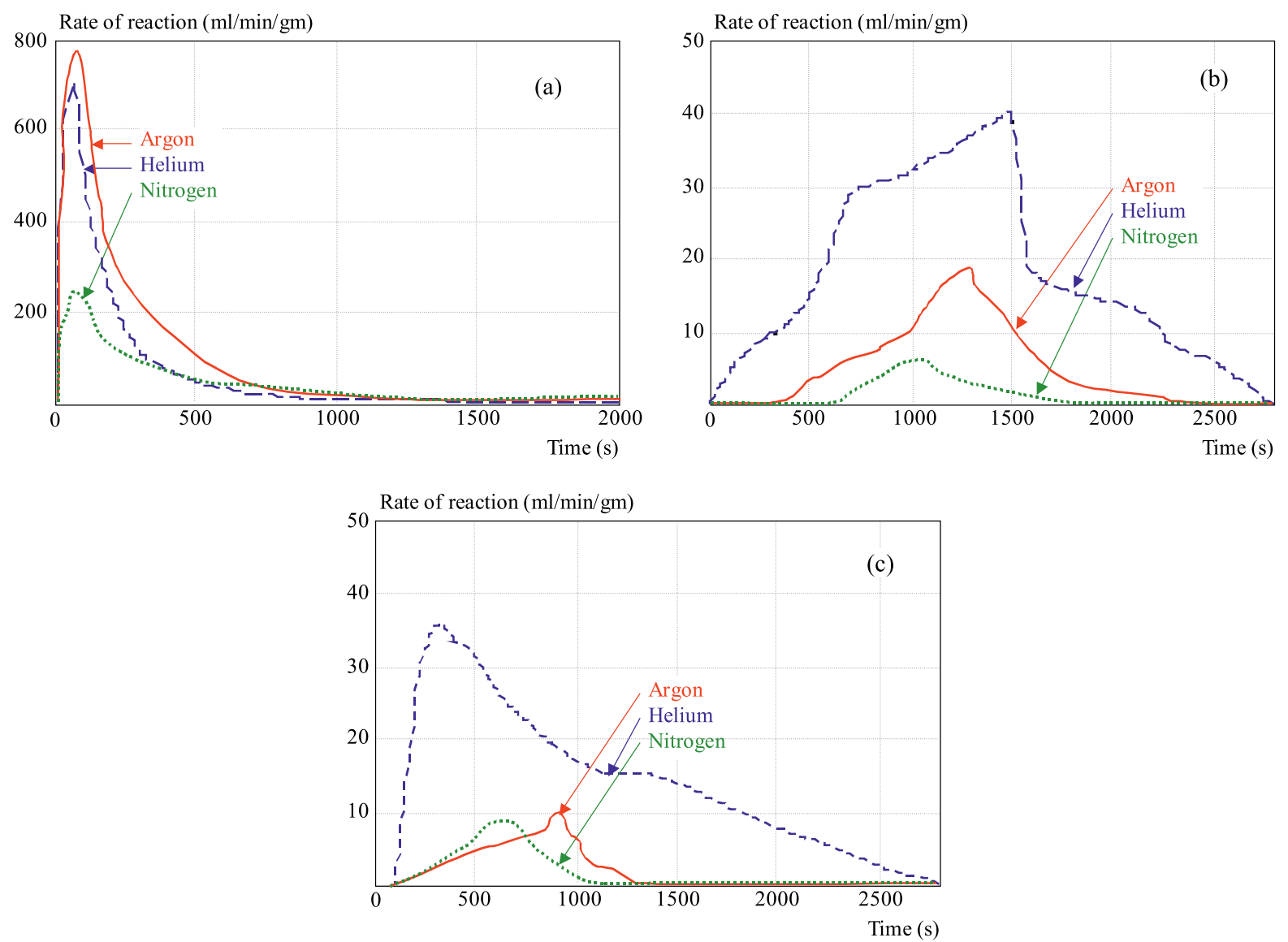

Fig. 8. Rate of reaction of nano aluminium particles produced in different ambience reacts with (a) $0.5 \mathrm{M} \mathrm{KOH}$ (b) water solution (c) water preheated to $70^{\circ} \mathrm{C}$

of reaction is due to the improved reactivity with the produced nano powders where surface area is high as compared to the commercial grade micron size aluminium powder. Ivanov et al, concluded that to facilitate continuous hydrogen generation, the metal particles of small size with high surface area are favourable [18].

Figure 7 shows the variation in rate of generation of hydrogen on reaction of nano aluminium powder generated in helium atmosphere with different molarity of potassium hydroxide solution. It is observed that the rate of reaction is high with increase in molarity of the $\mathrm{KOH}$ solution. In general it is observed that the rate of reduction in hydrogen generation after attaining the highest generation rate is almost the same with alkali solution of different molarity. The cause for it could be due to formation of alumina after reacting with water in the medium causing stabilised hydrogen generation and then on further reaction the rate gradually comes down to zero. Irrespective of molarity of $\mathrm{KOH}$, the reaction completes almost at the same time.

Figure 8 shows the rate of generation of hydrogen on reaction of nano aluminium powder (obtained from different atmospheric conditions with WEP) with potassium hydroxide and water solution. It is observed that the rate of hydrogen generation is high when the nano aluminium particle reacts with $\mathrm{KOH}$ solution compared to water. Also it is observed that rate of generation of hydrogen is more with the nano aluminium particles produced in argon ambience on its reaction with $\mathrm{KOH}$ compared with nano aluminium particles produced in helium/nitrogen ambience. It is to be mentioned that the reaction comes to an end almost in the same time irrespective of the particles generated from different ambience. The rate of hydrogen liberated is less with particles generated in nitrogen ambience. This could be due to the formation of AlN coating formed during the process of particle formation in wire explosion process in nitrogen ambience.

Figures $8 \mathrm{~b}$ and $\mathrm{c}$ show the variation in rate of generation of hydrogen on reaction of nano aluminium powder added to water at room temperature and with preheated water at $70{ }^{\circ} \mathrm{C}$ respectively. The nano particles generated in helium ambience liberate hydrogen for a longer duration. The amount of hydrogen generation has drastically decreased when added to the preheated water.

Generally Aluminium releases 3 electrons into the water and forms $\mathrm{Al}^{3+}$ ion. On its reaction in alkali solution, the reduction reaction occurs as producing hydrogen gas and hydroxide ions. The three $\mathrm{OH}^{-}$ions gets neutralised with $\mathrm{Al}^{3+}$ ions forming amorphous $\mathrm{Al}(\mathrm{OH})_{3}$. The hydrolysis reaction of AlN coated powder is slow compared to particles produced in Argon/Helium. Zhang et al [19] studied the hydrolysis process of surface treated aluminium nitride powders and concluded that hydrolysis product was amorphous species of $\mathrm{Al}(\mathrm{OH})_{3}$ until hydrol- 

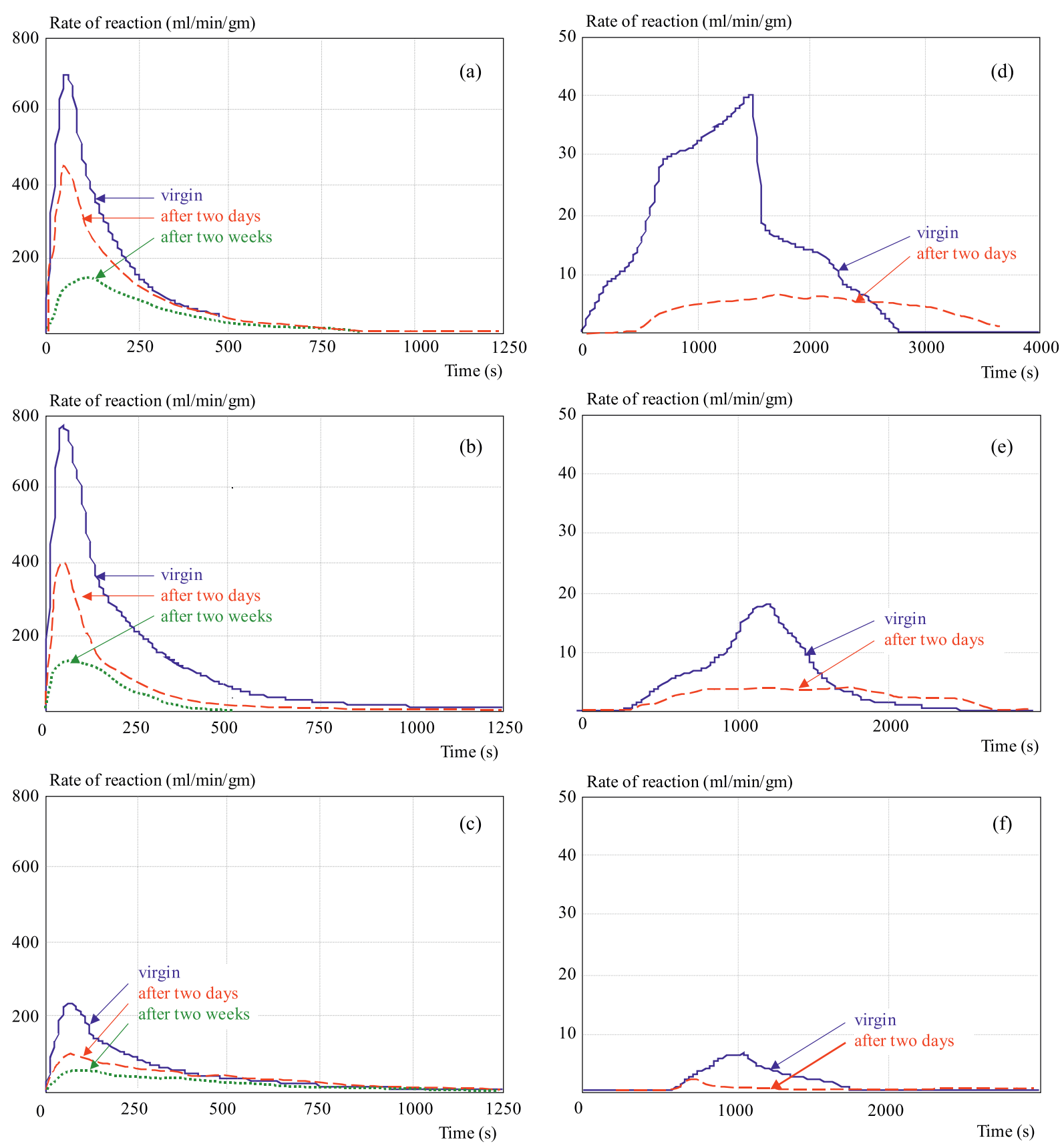

Fig. 9. Rate of reaction when nano aluminium particles are exposed to air medium and on its reaction with $0.5 \mathrm{M}$ KOH: (a) helium (b) argon and (c) nitrogen ambience, and water: (d) helium (e) argon and (f) nitrogen ambience

ysis time of 192 hours and becomes crystalline bayerite after 250 hours of hydrolysis.

$$
3 \mathrm{H}_{2} \mathrm{O}+3 \mathrm{e}^{-} \rightarrow \frac{3}{2} \mathrm{H}_{2} \uparrow+3 \mathrm{OH}^{-}
$$

Figure 9 shows the variation in generation of hydrogen for the nano particles exposed to atmospheric air for different time period and on its reaction with $\mathrm{KOH} /$ water solution. It is observed that there is drastic reduction in reaction rate for the powder exposed to air medium for longer duration, irrespective of the reactant water $/ \mathrm{KOH}$. The reduction in the rate of reaction occurs more with particles generated by WEP in argon ambience and is less with particles generated with helium and nitrogen ambience confirming that passivation of nano particles alters the fundamental characteristics of the nano particle. It is also observed that the nano aluminium particles produced in helium ambience in wire explosion process, generate hydrogen for longer duration when reacted with water compared with argon/nitrogen generated nano aluminium powder.

\section{CONCLUSIONS}

Nano-aluminium particles are produced through the wire explosion process in different gas medium. The median size of the particles produced by wire explosion pro- 
cess, in helium atmospheres is of smaller size compared to argon/nitrogen medium. The nano aluminium powder on reaction with water forms bayerite and boehmite structure, whereas on reaction with $\mathrm{KOH}$ solution at room temperature it forms bayerite. Also it is noticed that when the nano aluminium powder reacts with $\mathrm{KOH}$ maintained at $50{ }^{\circ} \mathrm{C}$, the oxide precipitates formed have both bayerite and boehmite structure. The reaction of nano aluminium powder with $\mathrm{KOH}$ solution/water indicates that the rate of hydrogen generation is high when the nano aluminium powder reacts with $\mathrm{KOH}$ solution than with water. The rate of hydrogen generation gets reduced drastically when the nano aluminium powder which is exposed to air medium for some period is used for reaction with $\mathrm{KOH} /$ Water. It is also observed that the rate of hydrogen generation is high with nano size aluminium particles compared with micron size particles. The result of the study confirms that thin layer of oxide/nitride formation in nano aluminium powder on reaction with $\mathrm{KOH} /$ Water shows reduction in rate of generation of hydrogen.

\section{Acknowledgement}

The author wish to thank Department of Science and Technology, New Delhi for sponsoring the project on generation of nano particles by wire explosion process.

\section{REFERENCES}

[1] KOTAY, S. M.-DAS, D.: Int. J. Hydrogen Energy 33 No. 1 (2008), 258.

[2] U.S. Department of Energy. Office of fossil energy Hydrogen Program Plan: Hydrogen from Natural Gas and Coal: the Road to a Sustainable Energy Future..

[3] De LUCA, L. T.-GALFETTI, L.-SEVERINI, F.-MEDA, L.-MARRA, G.-VOROZHTSOV, A. B.-SEDOI, V. S.BABUK, V. A.: Combustion, Explosion and Shock Waves 41 (2005), 680.

[4] KEARNS, M. : Material Science and Engineering A 375 (2004), 120.

[5] KIM, J. H.-LEE, H.-HAN, S. C.-KIM, H. S.-SONG, M. S.-LEE, J. Y.: J. Hydrogen Energy 29 (2004), 263.
[6] JIANG, V-YAtSui, K.: IEEE Trans. Plasma Science 26 (1998), 1498

[7] KWON, Y. S.-JUNG, Y. H.-YAVOROVSKY, N. A.-ILLYN, A. P.-KIM, J. S. : Scripta Mater 44 (2001), 2247.

[8] IVANOV, V.-KOTOV, Y. A.-SAMATOV, O. M.-BOHME, R.-KAROW, H. U.-SCHUMACHER, G. : Nanostructured Materials 6 (1995), 287.

[9] CHANDLER, K. M.-HAMMER, D. A.-SINARS, D. B.-PIKUZ, S. A.-SHELKOVENKO, T. A. : IEEE Transactions On Plasma Science 30 (2002), 577.

10] SEDOI, V. S.-MESYATS, G. A.-ORESHKIN, V. I.-VALEVICH, V.-CHEMEZOVA, L. I. : IEEE Trans. Plasma Science 27 (1999), 845.

11] SARATHI, R.-SINDHU, T. K.-CHAKRAVARTHY, S. R. : Materials Characterisation 58 (2007), 148.

12] HAYT, W. H.-KEMMERLY, J. E.-DURBIN, S. M.: Engineering Circuit Analysis, Sixth Edition, Tata Mcgraw-Hill, 2002.

13] SPOSitos, G.: The Environmental Chemistry of Aluminium, CRC Press, 1996.

14] SARATHI, R.-CHAKRAVARTHY, S. R.-VENKATASESHAIAH, C.: International Journal of Nanoscience 3 (2004), 819

[15] ZAKHARCHENYA, R. I.-VASILEVSKAYA, T. N. : J. Mater. Sci. 29 (1994), 2806.

Received 12 July 2009

Ramanujam Sarathi is currently professor and Head of high voltage laboratory, Department of electrical engineering, IIT Madras, Chennai, India. He obtained his Phd from Indian Institute of Science, Bangalore in 1994. His research areas includes condition monitoring of power apparatus and nano materials.

Binu Sankar is an research scholar working for his Phd program in the area of hydrogen generation. He completed his masters degree in the year 2004. He is an IEEE student member.

Satyanarayanan Raghuraman Chakravarthy is currently professor in the department of Aerospace engineering, IIT Madras, Chennai, India. He obtained his Phd from Georgia Institute of Technology in the year 1995. His research area includes nano materials and combustion engineering. 